\title{
Cortical plasticity catalyzed by prehabilitation enables extensive resection of brain tumors in eloquent areas
}

\author{
Paola A. Rivera-Rivera, MA, ${ }^{1}$ Marcos Rios-Lago, MA, PhD, ${ }^{4-6}$ Sandra Sanchez-Casarrubios, MA, \\ Osman Salazar, MD, ${ }^{1}$ Miguel Yus, MD, ${ }^{2}$ Mercedes González-Hidalgo, MD, PhD, ${ }^{3}$ \\ Ana Sanz, MA, PhD, ${ }^{1}$ Josué Avecillas-Chasin, MD, PhD, ${ }^{1}$ Juan Alvarez-Linera, MD, PhD, ${ }^{4}$ \\ Alvaro Pascual-Leone, MD, PhD, ${ }^{7,8}$ Antonio Oliviero, MD, PhD, ${ }^{9}$ and Juan A. Barcia, MD, PhD ${ }^{1}$
}

Departments of ${ }^{1}$ Neurosurgery, ${ }^{2}$ Radiology, and ${ }^{3}$ Neurophysiology, San Carlos Institute of Health Research (IdISSC), Hospital Clínico San Carlos de Madrid, Universidad Complutense de Madrid; ${ }^{2}$ Department of Radiology, Hospital Ruber Internacional, Madrid; ${ }^{5}$ Department of Basic Psychology II, UNED, Madrid; ${ }^{6}$ Brain Damage Unit, Hospital Beata María Ana, Madrid, Spain; ${ }^{7}$ Berenson-Allen Center for Noninvasive Brain Stimulation, Division of Cognitive Neurology, Department of Neurology, Beth Israel Deaconess Medical Center and Harvard Medical School, Boston, Massachusetts; 8 Institut Guttmann, Universitat Autonoma, Barcelona; and ${ }^{9}$ Department of Neurology and FENNSI group, Hospital Nacional de Parapléjicos, Toledo, Spain

OBJECTIVE The extent of resection is the most important prognostic factor following brain glioma surgery. However, eloquent areas within tumors limit the extent of resection and, thus, critically affect outcomes. The authors hypothesized that presurgical suppression of the eloquent areas within a tumor by continuous cortical electrical stimulation, coupled with appropriate behavioral training ("prehabilitation"), would induce plastic reorganization and enable a more extensive resection.

METHODS The authors report on 5 patients harboring gliomas involving eloquent brain areas within tumors as identified on intraoperative stimulation mapping. A grid of electrodes was placed over the residual tumor, and continuous cortical electrical stimulation was targeted to the functional areas. The stimulation intensity was adjusted daily to provoke a mild functional impairment while the function was intensively trained.

RESULTS The stimulation intensity required to impair function increased progressively in all patients, and all underwent another operation a mean of 33.6 days later (range 27-37 days), when the maximal stimulation voltage in all active contacts induced no functional deficit. In all cases, a substantially more extensive resection of the tumor was possible. Intraoperative mapping and functional MRI demonstrated a plastic reorganization, and most previously demonstrated eloquent areas within the tumor were silent, while there was new functional activation of brain areas in the same region or toward the contralateral hemisphere.

CONCLUSIONS Prehabilitation with continuous cortical electrical stimulation and appropriate behavioral training prior to surgery in patients with WHO Grade II and III gliomas affecting eloquent areas accelerate plastic changes. This can help maximize tumor resection and, thus, improve survival while maintaining function.

https://thejns.org/doi/abs/10.3171/2016.2.JNS152485

KEY WORDS glioma surgery; eloquent cortical areas; brain plasticity; cortical stimulation; preservation of brain functions; diagnostic and operative techniques; oncology; neurooncology

$\mathrm{T}$ HE most important prognostic factor of the survival duration following surgical treatment of gliomas, besides the histological grade, is the extent of resection..$^{11,20}$ The presence of eloquent areas within or near a tumor often limits resection, ${ }^{22}$ as resection performed in these areas would result in functional loss and thus affect the prognosis.

A way to increase the extent of resection, and thus improve the prognosis, would be to promote plastic changes by which brain areas distant from the glioma take over

ABBREVIATIONS CCES = continuous cortical electrical stimulation; $\mathrm{ECSM}=$ extraoperative cortical stimulation mapping; fMRI = functional MRI; ICSM = intraoperative cortical stimulation mapping; rTMS = repetitive transcranial magnetic stimulation; sMRI = structural MRI.

SUBMITTED October 27, 2015. ACCEPTED February 11, 2016.

INCLUDE WHEN CITING Published online May 20, 2016; DOI: 10.3171/2016.2.JNS152485. 
support for cognitive and behavioral functions of eloquent areas within the tumor. ${ }^{20}$ Low-grade gliomas can, as they grow slowly, induce such plasticity. ${ }^{19}$ We hypothesized that suppressing functional areas located within the tumor using continuous cortical electrical stimulation (cCES) while intensively training affected behaviors might catalyze cortical plasticity, accelerating plastic changes in a few weeks and leading other brain areas to take over support of the targeted behavior. If successful, such a preoperative "prehabilitation" process would not only enable a more extensive tumor resection, but it would also minimize postoperative functional impairment, improving prognosis while maintaining quality of life. Here, we present a study testing this hypothesis in 5 patients with WHO Grade II or III gliomas located in eloquent areas.

\section{Methods \\ Participants}

Five right-handed patients ( 3 males and 2 females) with a mean age of 41 years (range 27-52 years) with WHO Grade II or III (anaplastic) gliomas affecting eloquent areas of the left hemisphere gave written consent and entered the study, which was approved by the institutional ethics committee. All were native Spanish-language speakers, except for one patient who was proficient in Spanish, but whose mother tongue was Romanian (Table 1). All were free of a history of neurological or psychiatric disorders. Four patients had undergone a first operation several years before, but only a partial resection or biopsy of the tumor had been possible because functional areas were detected within it. In all cases, the tumor was growing, which caused progressing symptoms, or it had reached a large volume, suggesting a high risk of malignization. ${ }^{3}$

\section{Prehabilitation Protocol}

The prehabilitation protocol timeline is summarized in Fig. 1. Patients underwent brain structural MRI (sMRI) and functional MRI (fMRI), and neurological and neuropsychological assessment during the week prior to their first surgery. Tasks applied in the fMRI study were selected based on the patient's deficits (see Supplementary Table S1). The patients were hospitalized during the entire prehabilitation protocol. Awake surgery was performed under sedation (with propofol or dexmedetomidine), and during the surgery, cortical and subcortical intraoperative cortical stimulation mapping (ICSM) was performed (see the ICSM procedure in the Supplementary Material). The cortex was removed only if stimulation did not produce any deficit at a given site after 3 stimulations. The tumor was identified using the FLAIR signal by neuronavigation (StealthStation, Medtronic Inc.). In all cases, all or part of the tumor was not resected because functional areas were detected within it. A contoured subdural grid (AdTech Medical Instrument Corp. or Integra NeuroSciences Implants S.S.) was tailored to the desired shape and size, and placed covering the residual tumor to target functional areas within or immediately adjacent to it (median number of electrodes 48.2, range 32-64). After implantation, the grid was sutured to the dura mater, the dura and craniotomy were closed, leaving cables externalized, and the wound was closed. When brain edema had subsided (1 week after surgery), we performed extraoperative cortical stimulation mapping (ECSM) using electrical stimulation through the grid's electrodes to confirm the persistence of function in the same cortical areas mapped during surgery. Stimulation parameters were similar to those used during the ICSM procedure.

The day after the ECSM, the cCES period started. The grid's externalized cables were connected to external stimulators (Medtronic 3625 or 37022 screener, Medtronic Ibérica SA), and patients were subjected to cCES at a fixed $130-\mathrm{Hz}$ frequency and pulse width of $1 \mathrm{msec}$, aiming to disrupt and suppress cortical function. ${ }^{14}$ The stimulation intensity at each electrode contact pair was set daily to the threshold for the induction of a mild functional disturbance so that the patient could recover the corresponding disrupted function through daily intensive practice. The threshold was determined by increasing the stimulation intensity in increments of $0.5 \mathrm{~V}$ every 3 minutes while patients performed behavioral tasks. Cases 1,2, 3, and 5 performed different language tasks (object naming, repetitions of words, pseudowords and phrases, understanding simple and complex orders, and verbal fluency) under the supervision of a neuropsychologist and a speech therapist. Cases 4 and 5 practiced right shoulder movements (elevation, abduction, and flexion), right elbow movements (flexion, extension, pronation, and supination), and righthand fine motor movements (finger tapping, flexion and extension, abduction and adduction) under the supervision of a physiotherapist. In addition to the daily sessions of supervised training (formal training), during the rest of the day, patients were encouraged to continue unsupervised practice (self-applied training) except for planned rest periods, which included meals, sleep, and naps. cCES was continued nonstop for a median of 16 days (range 15-25 days) until it no longer resulted in functional deficits, despite maximal stimulation intensity (due to the limit of the stimulator used in Cases 1, 3, and 5; or patient tolerance in Cases 2 and 4; see Supplementary Fig. S3).

At this point, patients underwent a second ECSM procedure and fMRI following the same protocol used prior to the prehabilitation procedure, and a second surgery was planned. During the second surgery, intraoperative cortical stimulation mapping (ICSM) was performed according to the same parameters used during the first surgery, and all nonfunctional tumor tissue was removed following the same criteria and using the same technique as in the first surgery. Two to three months after the second surgery, patients underwent sMRI and a detailed neurological and neuropsychological assessment.

\section{Results \\ Case Descriptions}

Case 1

This case was previously reported. ${ }^{1}$ This 27 -year-old man harbored an anaplastic astrocytoma (WHO Grade III). Eighteen functional points that produced speech arrest, dysarthria, and dysnomia were identified during the first ICSM procedure.

In the first ECSM procedure, 12 pairs of grid electrodes 


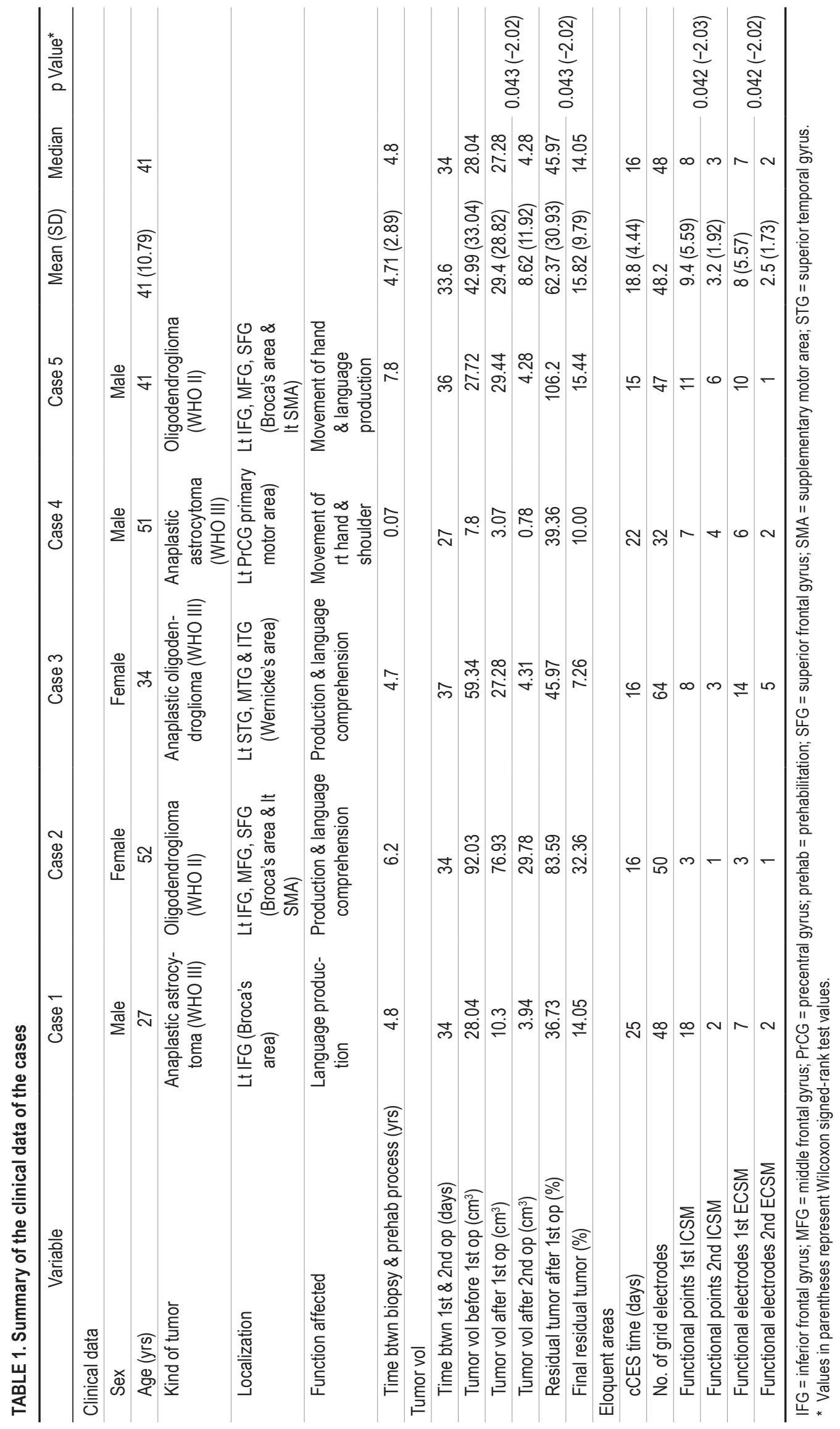




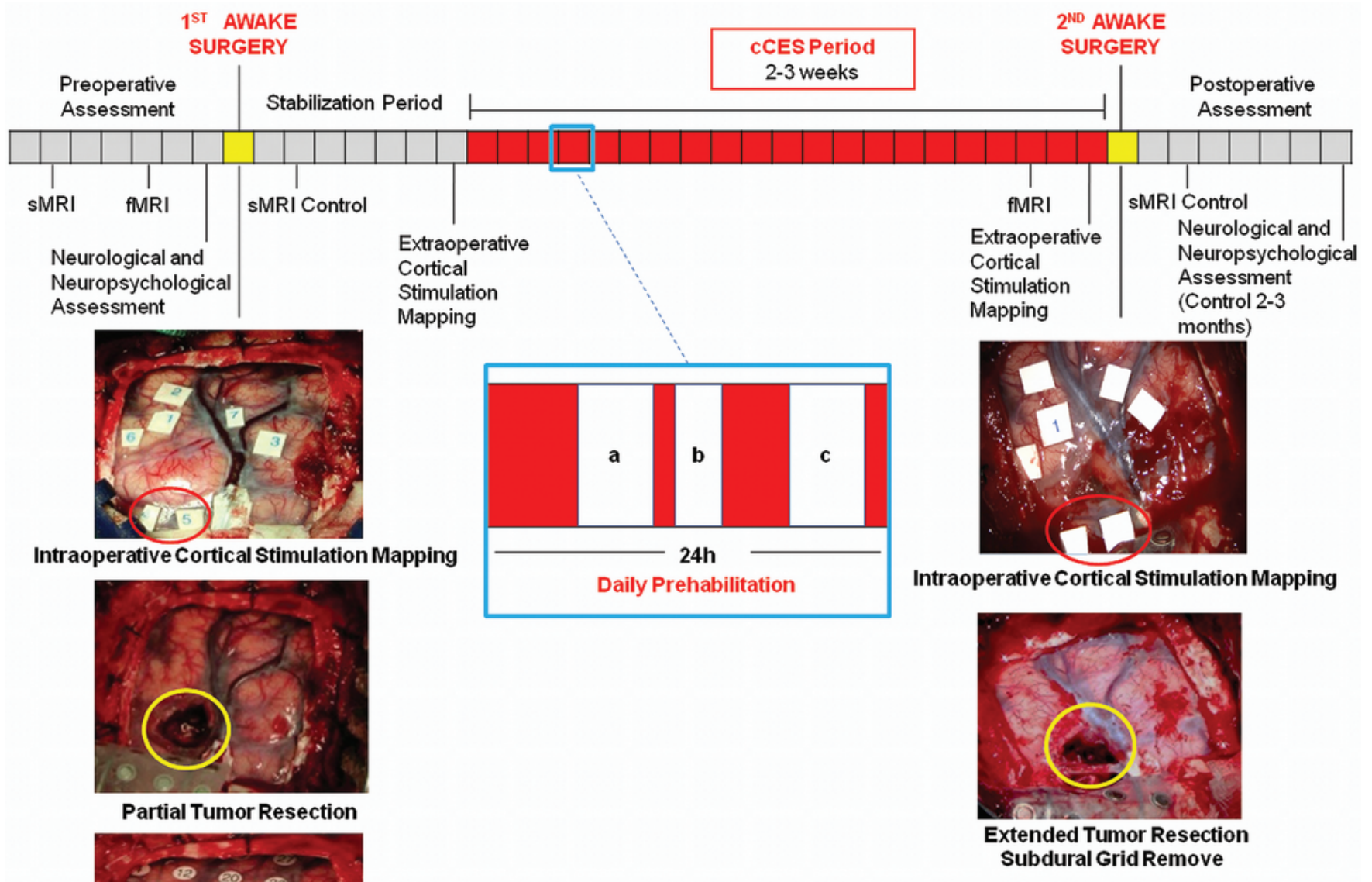

produced speech disturbances (speech arrest, dysarthria, and dysnomia). In the second ECSM procedure, 2 dysnomia points were identified, which were also detected in the second ICSM procedure. However, the deficits produced by stimulation of these 2 latter points disappeared after subcortical resection.

\section{Case 2}

This bilingual (Spanish and Romanian) 52-year-old woman had undergone a partial resection of a left frontal oligodendroglioma 6.2 years previously. The tumor progressed, and the fMRI study showed that it contained functional speech areas for Spanish and Romanian. One functional point for Spanish and 2 for Romanian language, which produced speech arrest, were identified during the first ICSM study. The first attempt to resect the tumor with awake surgery had to be stopped because of language deficits and behavioral changes (mainly disinhibition), leaving a partial resection, and a grid was implanted over the tumor. In the first ECSM study, 3 pairs of grid electrodes produced speech arrest, dysarthria, decreased verbal fluency, and dysnomia for Spanish language and speech arrest for Romanian language. cCES performed through the implanted cortical grid provoked a reorganization of the 2 language areas (Spanish and Romanian) at the basal aspect of the left inferior frontal gyrus, as seen on the second fMRI study. In the second ECSM study, all contacts were negative for Spanish, but the functional point for Romanian was present. This permitted a much wider resection of the tumor in a second awake surgery. The pa- 
tient suffered from a supplementary motor area syndrome during surgery, with complete right hemiplegia and aphasia. She recovered completely within 2 weeks, except for a mild fluency impairment, but useful language remained. This patient showed a $10 \%$ decrease in the postsurgical language comprehension assessment.

\section{Case 3}

This 34-year-old woman harbored an anaplastic oligodendroglioma in the superior left temporal gyrus, affecting Wernicke's area. She had undergone surgery 4.7 years previously, with partial removal of the tumor. In the first awake surgery, the ICSM study showed 8 functional points that produced dysnomia, perseverance, and paresthesia in the right hand. Thus, only a standard left temporal lobectomy could be achieved, and a grid was implanted. In the first ECSM study 14 pairs of grid electrodes produced dysnomia, alexia, failure in reading comprehension, right hand paresthesias, contraction of the right hemiface, and speech arrest. The fMRI study obtained after the prehabilitation process suggested a shift of the comprehension function of language from the left to the right side. In the second ECSM study, there were 5 functional points: 1 functional point provoking paresthesia in the right hand, 1 provoking right facial hemiclonus, and 3 points inducing paraphasias, dysnomia, decreased fluency, and dysphasia. The remaining 9 contacts previously provoking dysnomia and alexia did not produce any effect. This permitted a much wider tumor resection in a second awake surgery.

\section{Case 4}

This 51-year-old man presented with focal motor seizures in the right hand, followed by a mild paresis (Grade $4 / 5$ according to the Oxford Scale) of the fingers of the right hand. The sMRI study suggested a low-grade glioma located in the left precentral gyrus. The fMRI showed the motor function of the hand located beside and within the tumor. The patient underwent awake surgery 25 days after diagnosis, where a partial reorganization of functions around the tumor was found.

During this first ICSM study, 7 functional points that produced ulnar flexion of the wrist, arm extension, forearm pronation, pharyngeal musculature contraction, and extension of the right index finger were identified, but a complete resection could be not be performed because hand and shoulder motor functions were detected within the tumor. After this, the patient presented with light paresthesias in the right distal fingers, but during the following 14 hours, these paresthesias recovered. A cortical electrode grid was implanted over the tumor remnant. The control MRI study showed a partial resection. The histopathological diagnosis was an anaplastic astrocytoma.

In the first ECSM study, 6 pairs of grid electrodes produced paresthesias in the right hand and hemithorax, arm flexion, tongue contraction, and shoulder elevation. The patient underwent the prehabilitation protocol, and the second ECSM study showed that 2 pairs of electrodes were still active: one produced interference with forearm flexion and shoulder elevation and the other one facial hemiclonus. The fMRI study performed before the second surgery exhibited displacement of motor functions to the depth of the central sulcus. When the patient underwent reoperation, the second ICSM study showed 4 functional points with mild paresthesias in the right hand and fingers, and shoulder elevation was identified around the tumor. These functions previously located over the tumor had cleared (i.e., they were no longer functional), permitting an almost complete tumor removal. During removal, there was an immediate deficit in shoulder movement, but a few hours after surgery, the patient recovered shoulder mobility.

\section{Case 5}

This 41-year-old man had been diagnosed with a Grade II left frontal oligodendroglioma covering motor movement and language areas 7.8 years earlier. Only a biopsy could be performed at that time, because a complete aphasia occurred during surgery.

The tumor extended from the left precentral gyrus to the left inferior frontal gyrus. The fMRI study showed the presence of language production and movement areas within the tumor. During the first ICSM procedure, 11 functional points that produced speech arrest, dysarthria, dysnomia, and impairment in tapping were identified. At the first removal attempt, the presence of language areas within the tumor led to a complete aphasia when resection was attempted. A subdural grid was implanted, but the bone flap was not replaced because of edema at the tumoral and peritumoral areas.

The stabilization period after first awake surgery was complicated by the appearance of a subdural hematoma over the cortical grid. This was removed emergently, leaving residual right hemiplegia and aphasia, which resolved in 1 week but resulted in a mild fluent dysphasia. In the first ECSM study, 10 pairs of grid electrodes produced speech arrest, dysnomia, neologisms, tongue paresthesias, impairment in movements of the right hand and fingers, and semantic paraphasia. The prehabilitation period was initiated after the patient's recovery by intensive speech and physical rehabilitation. The fMRI study obtained after the prehabilitation period showed reorganization of the language production and motor hand areas.

During the second ECSM study, stimulation of 1 pair of electrodes still produced phonological paraphasias, whereas the remaining 9 electrode pairs were did not produce any effect. In the second ICSM study, 6 functional points were identified. Three of them produced speech arrest and the other 3 produced mild paresthesias in the right fingers and lips. The sMRI study obtained after the second surgery showed an almost complete tumor resection. This patient, whose verbal fluency was below the 2 nd percentile at baseline, showed a worsening in performance during the postoperative evaluation.

\section{Complications}

All patients tolerated the prehabilitation protocol. Patients were receiving levetiracetam $(500 \mathrm{mg} / 12$ hours). Three patients (Cases 1, 4, and 5) experienced focal seizures that were controlled with medication and changes in the stimulation intensity. The stimulation intensity was lowered for less than 1 hour, and diazepam was given through a previously placed intravenous line until the symptoms disappeared. Cortical stimulation was then 
continued uninterruptedly until the next day's threshold setting session. The patient in Case 4 experienced occasional myoclonus in the right index finger, which produced no disability. One patient (Case 5) experienced 1 isolated seizure 8 months after the second surgery.

Two patients developed infections. In Case 1 there was osteomyelitis of the bone flap, and it was removed. In Case 3 , an epidural abscess associated with transitory worsening of motor and language functions was evacuated.

In Case 5, a subdural hematoma forming in the immediate postoperative period of the first surgery, over the cortical grid, provoked a complete motor aphasia and a paralysis $(0 / 5)$ of the right arm and face and paresis (1/5) of the right leg, and had to be surgically evacuated. The patient recovered almost complete motor function in the following days, but mild dysphasia persisted afterward. This patient had to undergo reoperation 1 year after the second surgery due to pseudomeningocele.

The patients in Cases 3 and 5 suffered deterioration of their language functions in the long term. The patient in Case 3 exhibited slight alteration in understanding and slowing of information processing. One patient (Case 5) experienced a slight motor aphasia. Another patient (Case 1) suffered a rapid tumor progression. The tumor transformed into a glioblastoma, and the patient died of the disease 8 months after the first operation.

\section{Induction of Cortical Plasticity}

In all 5 patients, the prehabilitation protocol resulted in the displacement of eloquent areas within the tumor, as demonstrated by ICSM and ECSM (Fig. 2 and Table 1). Furthermore, in all cases, fMRI showed the suppression of activation of previously activated eloquent areas in the stimulated cortex and the appearance of activation in other areas (Fig. 3), either within the same hemisphere or in the contralateral hemisphere (see the Methods for details; fMRI analyses are shown in the Supplementary Methods).
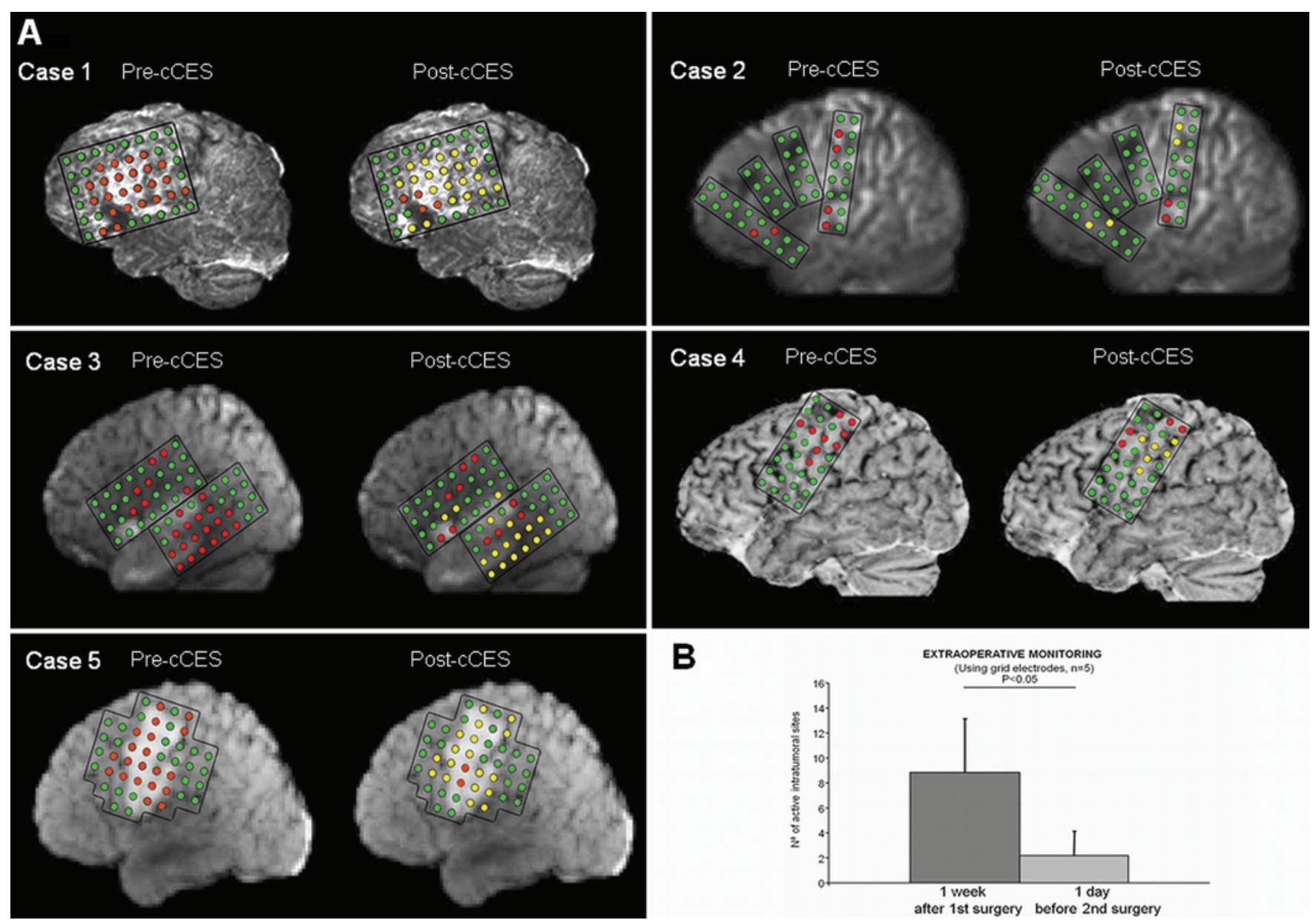

B

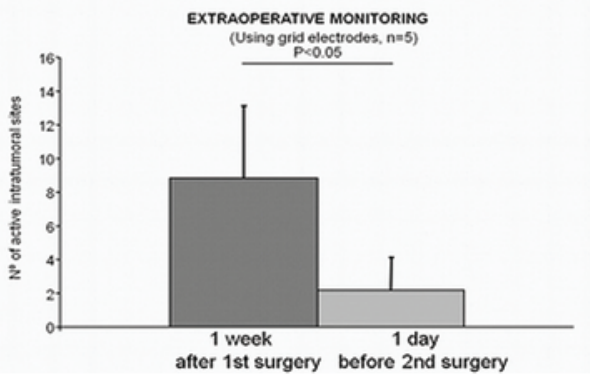

FIG. 2. Changes in active contacts before and after the prehabilitation procedure. A: ECSM. Variations before and after the CCES procedure in active contacts during the 2 ECSM procedures in the 5 patients. Images are surface renderings of the FLAIR sequence MR image after the first surgery. The contours of the grids and contacts are illustrative of their location after the grid implant. Red indicates contacts that produce a functional effect during the first or second test; yellow, contacts that produce a functional arrest during the first, but not the second test; and green, contacts that produce no functional arrest during the first and second test. B: Extraoperative monitoring. The graph shows the number of sites within the tumor that were identified as supporting some speech or motor function. After a few weeks of treatment (combined cCES and the prehabilitation procedure), the number of active sites was reduced (Wilcoxon test, $p<0.05$ ). Bars represent the median and the whiskers the quartiles. 

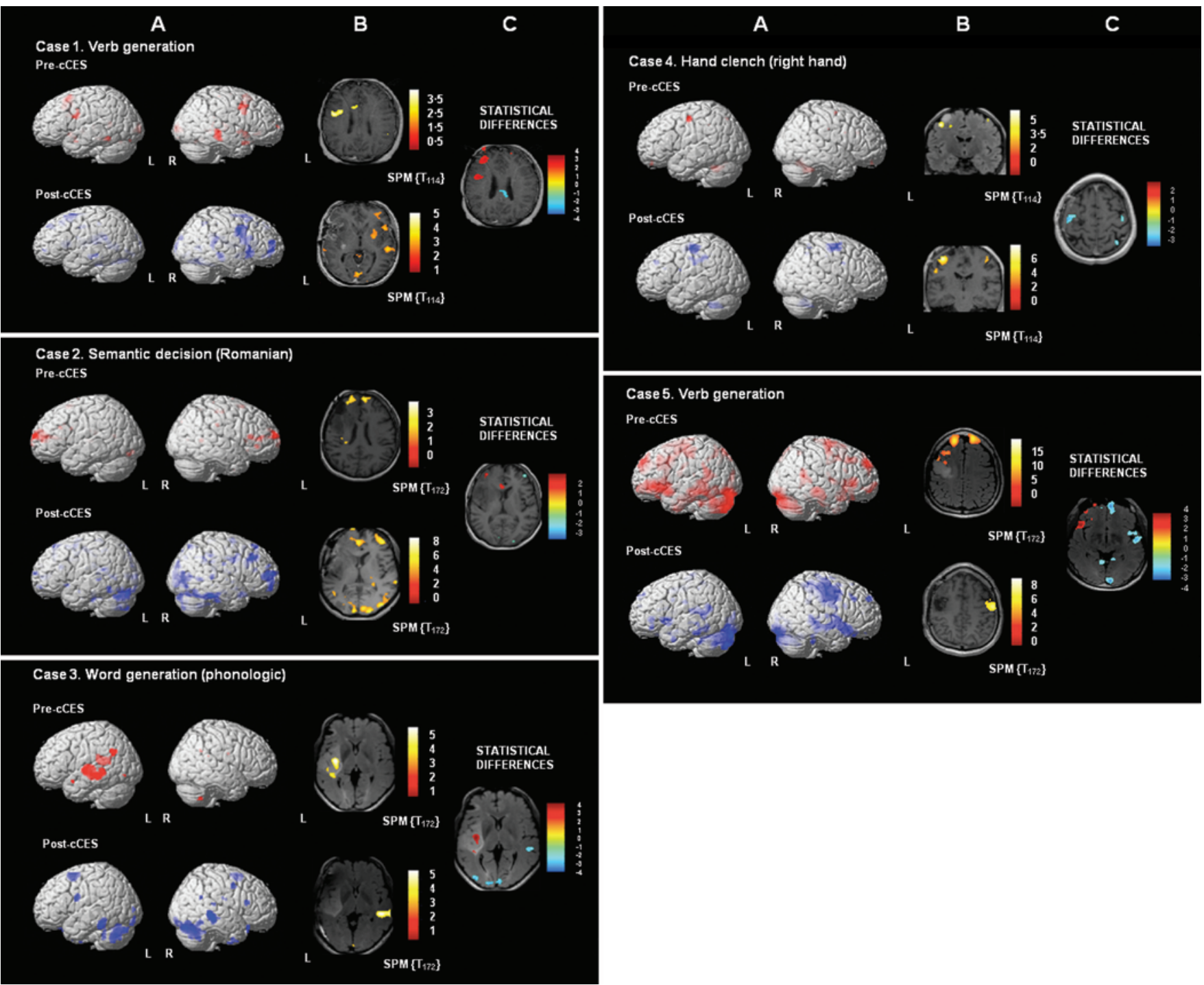

FIG. 3. Cortical reorganization. T-contrast maps showing activated regions for each case in one of the paradigms employed. A: Right and left views of the brain show the pre-cCES (activation shown in red) and post-cCES (activation shown in blue). Statistical maps were overlaid on the Colin Holmes 27 (ch2) template of the International Consortium for Brain Mapping (ICBM). B: Representative slices are shown for every case. Statistical maps are superimposed on the anatomical MR image obtained for each case. Neurological convention is followed (right = right). C: T-contrast maps showing significant differences for both contrasts: pre > post (shown in red) and post > pre (shown in blue). Neurological convention is followed $(R=R)$.

\section{Extent of Tumor Resection}

In all cases, the resection volume obtained during the second surgery, estimated using structural MRI (sMRI), was greater than the one obtained in the first surgery, despite identical surgical technique and criteria (Fig. 4 and Table 1). The mean tumor volume before the first surgery was $42.99 \pm 33.04 \mathrm{~cm}^{3}$. The mean tumor volume after first surgery was $29.4 \pm 28.82 \mathrm{~cm}^{3}$ while the mean tumor volume after second surgery was $8.62 \pm 11.92 \mathrm{~cm}^{3}$. The final residual tumor percent was $15.82 \% \pm 9.79 \%$, less than 30 $\mathrm{cm}^{3}$ in all cases.

\section{Neuropsychological Assessment}

Follow-up assessment 2-3 months after the second surgery showed that naming ability, measured using the Bos- ton naming test, ${ }^{18}$ improved or was maintained in all cases. Language comprehension, measured with the Token Test, ${ }^{5}$ was maintained or improved in all cases, except in Case 2. Similar results were found for verbal fluency tasks in the 3 patients tested for them, and who showed maintenance of the performance. Motor performance was also preserved in all cases. Neuropsychological assessment of other cognitive processes that were not part of the prehabilitation protocol showed a slight worsening in Cases 2, 4, and 5, for memory, executive function, and attention (see Supplementary Tables S2 and S3).

\section{Discussion}

We present a case series of 5 patients with gliomas af- 


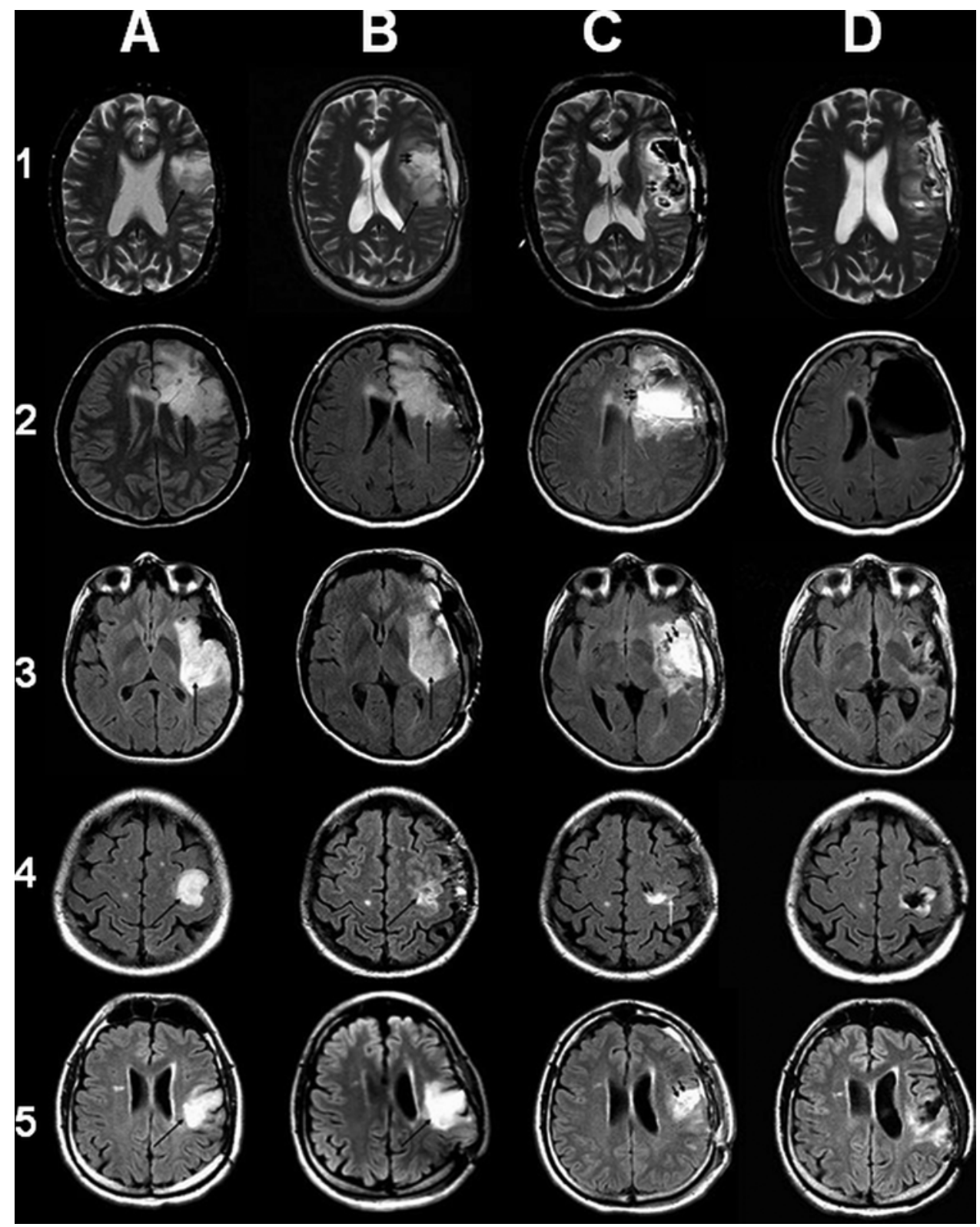

FIG. 4. Tumor volumes. Magnetic resonance images of the cases obtained before the first surgery (A), immediately after the first surgery (B), immediately after the prehabilitation procedure and the second surgery (C), and more than 1 month after the second surgery (D). Sequences are T2-weighted for Case 1 and FLAIR for Cases 2 through 5. Note that in the FLAIR sequences, the tumor is shown as having a medium-high intensity (single large arrow), and the immediate postoperative resection cavity with serohematic content is shown as having a bright, high intensity (double short arrows).

fecting eloquent brain areas who underwent a prehabilitation protocol that catalyzed the plastic relocation of cortical functions away from the tumor. This enabled more extensive resections, without disabling functional deficits, than achievable using current state-of-the-art neurosurgical procedures. These findings confirm and critically extend the results of a successful proof-of-principle previously reported study in a single patient. ${ }^{1}$

The prehabilitation protocol, combining continuous cortical electrical stimulation with intensive behavioral training, resulted in a remapping of function in all our patients' brains over the course of only a few weeks. At the end of the prehabilitation period: 1) intraoperative cortical stimulation mapping (ICSM) no longer disrupted neurological function when stimulating most of the same regions initially identified as eloquent cortex; 2) the fMRI showed a change in the brain areas previously activated during the task; and 3) more extensive resection of the tumors was possible, including brain regions identified as eloquent cortex during the initial ICSM, prior to prehabilitation, leading to minimal functional deficits.

ICSM is the gold standard for resecting gliomas in eloquent areas, as it allows the identification of brain regions that lead to symptoms when disrupted by stimulation, in- 
dicating that their resection is likely to result in a longlasting disability. ${ }^{21}$ Nonetheless, in some instances, deficits after resection of ICSM-identified eloquent areas may be followed by some functional compensation weeks later. ${ }^{6}$ Low-grade gliomas have also been previously described to induce topographic cortical plasticity, ${ }_{1}^{13}$ permitting a multistage resection of these tumors several years after the first surgery.$^{19}$ However, in all 5 of our patients, a second, more extensive surgery was possible after a few weeks, suggesting that the prehabilitation protocol accelerated the underlying neurobiological process.

We first attempted to induce the reported plastic brain reorganization in a patient with a left-sided precentral oligodendroglioma, which could not be completely removed because it affected language areas. We applied 12 daily sessions of repetitive transcranial magnetic stimulation (rTMS). Although the functional and behavioral impact of the daily rTMS sessions progressively decreased, possibly suggesting plastic changes, these did not lead to a functional remapping and they did not enable a more extensive resection. ${ }^{2}$ In a subsequent case (Case 1 of this study), to achieve a stronger and more sustained disruption of the eloquent cortex within a tumor, we applied direct cortical stimulation for 25 days, using a frequency of $130 \mathrm{~Hz}$ and a pulse width of $1 \mathrm{msec}$, and setting the stimulation intensity to the threshold at which a mild speech disturbance was evident without any other neurological effects. ${ }^{1}$ This treatment successfully achieved the displacement of speech functions, and a more radical resection of the tumor was possible in a second surgery. In the present case series, we expand on this proof-of-principle study by demonstrating that similar effects can be reproducibly achieved in patients with language or motor involvement, each requiring only few weeks of prehabilitation.

Cortical stimulation may suppress function in the targeted regions, promoting plastic changes that are induced by the demand imposed by the training. In our prehabilitation protocol, continuous stimulation was applied with the aim of disrupting cortical functions in eloquent brain regions within the glioma, while patients also underwent intensive behavioral training. We suspect that both the continuous disruption of the function by cCES and the intensive training of the function are necessary. Nonetheless, the relative role of cCES versus behavioral training in the observed cortical reorganization remains unclear and warrants further study. Future work should also evaluate the neurological effects of cCES and behavioral training independently.

We hypothesized that the cortical disruption induced by cCES had to impair, but still permit, function, and thus the cCES intensity was adjusted daily to maximize its functional impact. Future studies are needed to explore the optimal cCES parameters.

In all of our patients, fMRI revealed the activation of brain areas during the execution of the affected functions that were inactive before the prehabilitation process. This might reflect paradoxical functional facilitation, ${ }^{9}$ reminiscent of the improvement in language or motor recovery by disrupting the unaffected hemisphere with the aid of noninvasive brain stimulation in patients after a stroke..$^{10}$ The rapid time course suggests shifts in strength or the un- masking of existing connections that may secondarily lead to more stable structural changes. ${ }^{16}$ However, we speculate that the involved mechanism is not the immediate release of parallel networks, ${ }^{7}$ but a reorganization of preexisting networks during the prehabilitation process. Stimulation at higher intensities than the threshold level led to a complete loss of function in the mapping performed at the initial surgery, but not in the one performed following the prehabilitation process. The transition between the 2 stable states may not be possible spontaneously, but may instead require catalysis by prehabilitation. It has been shown that training induces changes in the cortical representation of functions (e.g., finger movements) that are reversible for a short period, but are stable after a time span of 5 weeks, similar to our prehabilitation process. ${ }^{17}$ Additionally, electrical stimulation has been shown to promote structural changes in animal studies., ${ }^{423}$ Whether the changes in our cases are due to synaptic facilitation or to structural changes, including dendritic arborization and synaptogenesis, should be determined using experimental models. However, the slow growth of the tumor could have previously induced a plastic change, which was completed by the prehabilitation process. Also, the first surgery could have provoked a delayed reorganization through neurogenetic or plastic mechanisms, a question which cannot be ruled out due to the lack of controls. Furthermore, the fact that 4 of the 5 patients did undergo prior surgeries involving the functional areas could constitute another confounder factor.

In all of our patients, overall language and motor function were maintained at a clinically useful level (permitting autonomous daily life and sufficient communication abilities) after the second surgery. In 2 patients (Cases 1 and 4), the prehabilitation-trained function actually improved after surgery, and this improvement was sustained. In Cases 3 and 5, the language functions improved during the prehabilitation period and after surgery, but there was some deterioration after a period of follow-up. In both of these cases, the resection was smaller than the cCES area. We speculate that eloquent areas around the tumor that were spared in the second surgery may interfere with remapped areas when they were no longer stimulated, thus reversing some of the plastic changes induced by the prehabilitation. Nonetheless, even in such instances, we believe that the intervention was useful. Malignant transformation risk in gliomas is related to residual tumor volume, with a critical threshold suggested to be $30 \mathrm{~cm}^{3}$. Without our prehabilitation protocol, the residual volume was $30 \mathrm{~cm}^{3}$ in 2 cases (Cases 2 and 3) and near $30 \mathrm{~cm}^{3}$ in 2 other cases (Cases 1 and 5). After prehabilitation, the final residual volume percent was $15.82 \% \pm 9.79 \%$, less than $30 \mathrm{~cm}^{3}$ in all cases. Total resection was not achieved in any case, perhaps because the stimulative disruption was applied only to the tumoral areas and not to the peritumoral ones, with a supramarginal resection intent. Also subcortical white matter tracts en passage could have not been influenced by the stimulation of only the cortical areas.

The complication rate was unusually high. The main reason for complications in our patients was the relatively long period of implantation of subdural electrodes with externalized electrodes. Improvements in the connection to external stimulators or implantable stimulators could help 
minimize complications. To avoid other surgical complications, noninvasive stimulation procedures should also be further explored. Studies of functional connectivity, which can be altered in patients with brain tumors ${ }^{15}$ as part of the planning of the intervention, could be valuable to predict the possibility of enhancing the demonstrated effect by individually tailoring the intervention and defining the site of stimulation on the basis of the identified connectivity. ${ }^{8}$

Although therapeutic brain stimulation is widely used, ${ }^{14}$ there are no reports of tumorigenic side effects, and, in fact, electrical stimulation may slow down the growth of some brain tumors. ${ }^{12}$ However, further careful evaluation of the safety of the proposed technique is required.

\section{Conclusions}

We present a study of 5 patients with WHO Grade II and III gliomas located in the eloquent cortex who underwent a novel prehabilitation procedure that combined cCES with appropriate intensive behavioral training. This protocol enabled a more extensive tumor resection while preserving behavioral and cognitive functions. It is necessary to reproduce these results in a larger number of patients, with a longer follow-up, and to reduce the complication rate before prehabilitation can be widely used to improve the prognosis and survival of patients with glioma, while preserving their quality of life.

\section{Acknowledgments}

We thank Dr. Juan Raul Brin, Dr. Fernando Rascón (Department of Neurosurgery), Dr. Pedro Pérez Segura (Department of Oncology), Dr. Manuela Jorquera (Department of Radiology), Dr. Idoya Barca, Mrs. Carmen De Las Heras (Department of Rehabilitation), and the nursing team of the ward and operating room of the Hospital Clínico San Carlos for their technical help. We are indebted to the patients and their families for agreeing to participate in this trial. We thank Drs. Andrés Lozano, Alfredo QuiñonesHinojosa, Nader Sanai, and Mitchell Berger for critically reading the manuscript and for their useful comments.

This work was partly supported by a grant from the Fondo de Investigaciones Sanitarias of the Spanish Government FIS PI13/00512.

\section{References}

1. Barcia JA, Sanz A, Balugo P, Alonso-Lera P, Brin JR, Yus M, et al: High-frequency cortical subdural stimulation enhanced plasticity in surgery of a tumor in Broca's area. Neuroreport 23:304-309, 2012

2. Barcia JA, Sanz A, González-Hidalgo M, de Las Heras C, Alonso-Lera P, Díaz P, et al: rTMS stimulation to induce plastic changes at the language motor area in a patient with a left recidivant brain tumor affecting Broca's area. Neurocase 18:132-138, 2012

3. Berger MS, Deliganis AV, Dobbins J, Keles GE: The effect of extent of resection on recurrence in patients with low grade cerebral hemisphere gliomas. Cancer 74:1784-1791, 1994

4. Cooperrider J, Furmaga H, Plow E, Park HJ, Chen Z, Kidd $\mathrm{G}$, et al: Chronic deep cerebellar stimulation promotes longterm potentiation, microstructural plasticity, and reorganization of perilesional cortical representation in a rodent model. J Neurosci 34:9040-9050, 2014

5. De Renzi E, Vignolo LA: The token test: A sensitive test to detect receptive disturbances in aphasics. Brain 85:665-678, 1962
6. Duffau H, Capelle L, Denvil D, Sichez N, Gatignol P, Lopes $\mathrm{M}$, et al: Functional recovery after surgical resection of low grade gliomas in eloquent brain: hypothesis of brain compensation. J Neurol Neurosurg Psychiatry 74:901-907, 2003

7. Duffau H: New concepts in surgery of WHO grade II gliomas: functional brain mapping, connectionism and plasticity-a review. J Neurooncol 79:77-115, 2006

8. Fox MD, Halko MA, Eldaief MC, Pascual-Leone A: Measuring and manipulating brain connectivity with resting state functional connectivity magnetic resonance imaging (fcMRI) and transcranial magnetic stimulation (TMS). Neuroimage 62:2232-2243, 2012

9. Kapur N: Paradoxical functional facilitation in brain-behaviour research. A critical review. Brain 119:1775-1790, 1996

10. Kapur N, Cole J, Manly T: The paradoxical brain. Psychologist 26:102-105, 2013

11. Keles GE, Lamborn KR, Berger MS: Low-grade hemispheric gliomas in adults: a critical review of extent of resection as a factor influencing outcome. J Neurosurg 95:735-745, 2001

12. Kirson ED, Dbalý V, Tovarys F, Vymazal J, Soustiel JF, Itzhaki A, et al: Alternating electric fields arrest cell proliferation in animal tumor models and human brain tumors. Proc Natl Acad Sci U S A 104:10152-10157, 2007

13. Krieg SM, Sollmann N, Hauck T, Ille S, Foerschler A, Meyer $\mathrm{B}$, et al: Functional language shift to the right hemisphere in patients with language-eloquent brain tumors. PLoS One 8:e75403, 2013

14. Lozano AM, Lipsman N: Probing and regulating dysfunctional circuits using deep brain stimulation. Neuron 77:406424, 2013

15. Martino J, Honma SM, Findlay AM, Guggisberg AG, Owen JP, Kirsch HE, et al: Resting functional connectivity in patients with brain tumors in eloquent areas. Ann Neurol 69:521-532, 2011

16. Pascual-Leone A, Amedi A, Fregni F, Merabet LB: The plastic human brain cortex. Annu Rev Neurosci 28:377-401, 2005

17. Pascual-Leone A, Nguyet D, Cohen LG, Brasil-Neto JP, Cammarota A, Hallett M: Modulation of muscle responses evoked by transcranial magnetic stimulation during the acquisition of new fine motor skills. J Neurophysiol 74:10371045, 1995

18. Peña-Casanova J, Quiñones-Ubeda S, Gramunt-Fombuena N, Aguilar M, Casas L, Molinuevo JL, et al: Spanish Multicenter Normative Studies (NEURONORMA Project): norms for Boston naming test and token test. Arch Clin Neuropsychol 24:343-354, 2009

19. Robles SG, Gatignol P, Lehéricy S, Duffau H: Long-term brain plasticity allowing a multistage surgical approach to World Health Organization Grade II gliomas in eloquent areas. J Neurosurg 109:615-624, 2008

20. Sanai N, Berger MS: Glioma extent of resection and its impact on patient outcome. Neurosurgery 62:753-764, 264266, 2008

21. Sanai N, Berger MS: Recent surgical management of gliomas. Adv Exp Med Biol 746:12-25, 2012

22. Sanai N, Mirzadeh Z, Berger MS: Functional outcome after language mapping for glioma resection. N Engl J Med 358:18-27, 2008

23. Zheng J, Liu L, Xue X, Li H, Wang S, Cao Y, et al: Cortical electrical stimulation promotes neuronal plasticity in the peri-ischemic cortex and contralesional anterior horn of cervical spinal cord in a rat model of focal cerebral ischemia. Brain Res 1504:25-34, 2013

\section{Disclosures}

Dr. Pascual-Leone reports that he serves on the scientific advisory boards for Nexstim, Neuronic, Starlab Neuroscience, Neu- 
roelectrics, Axilum Robotics, Magstim Inc., and Neosync, and is listed as an inventor on several issued and pending patents on the real-time integration of TMS with electroencephalography and MRI.

\section{Author Contributions}

Conception and design: Barcia. Acquisition of data: Barcia, Rivera-Rivera, Rios-Lago, Sanchez-Casarrubios, Salazar, Yus, González-Hidalgo, Sanz, Alvarez-Linera. Analysis and interpretation of data: Barcia, Rivera-Rivera, Rios-Lago, SanchezCasarrubios, Salazar, Yus, Oliviero. Drafting the article: Barcia, Rivera-Rivera, Rios-Lago. Critically revising the article: Barcia, Rios-Lago, Pascual-Leone, Oliviero. Reviewed submitted version of manuscript: all authors. Approved the final version of the manuscript on behalf of all authors: Barcia. Statistical analysis: Rios-Lago, Yus, Oliviero. Administrative/technical/material sup- port: Barcia, Rivera-Rivera, Salazar, Yus, González-Hidalgo, Sanz, Avecillas-Chasin. Study supervision: Barcia.

\section{Supplemental Information}

Online-Only Content

Supplemental material is available with the online version of the article.

Supplementary Materials. http://thejns.org/doi/suppl/10.3171/ 2016.2.JNS152485.

\section{Correspondence}

Juan A. Barcia, Department of Neurosurgery, Hospital Clínico San Carlos de Madrid, Universidad Complutense de Madrid, Sc/Profesor Martin Lagos s/n, Madrid 28040, Spain. email: jabarcia@ucm.es. 